\title{
Phase responses to light pulses in mice lacking functional per or cry genes
}

\author{
K.Spoelstra ${ }^{* 1}$, U. Albrecht ${ }^{\dagger}$, G. T. J. van der Horst ${ }^{\ddagger}$, V. Brauer $^{*}$ and S.Daan* \\ *Zoological Laboratory, University of Groningen, Haren, the Netherlands \\ ${ }^{\dagger}$ Department of Biochemistry, University of Fribourg, CH-1700 Fribourg, Switzerland \\ ${ }^{\ddagger}$ MGC, Department of Cell Biology and Genetics, Erasmus MC, PO Box 1738, 3000 DR \\ Rotterdam, the Netherlands
}

submitted: Journal of Biological Rhythms

revised version June 8, 2004

1. To whom all correspondence should be addressed: Biological Center, University of Groningen, Kerklaan 309751 NN, Haren, The Netherlands

*Address for correspondence and proofs: Ir. K. Spoelstra, Zoological Laboratory, Kerklaan 30, 9751 NN Haren (Groningen), The Netherlands; e-mail K.Spoelstra@biol.rug.nl.

36 pages, 1 table, 8 figures. 


\section{Abstract}

The phase resetting properties of the circadian system in mice with a functional deletion in either $m C r y 1, m C r y 2, m P e r 1$ or $m P e r 2$ were studied in two experiments. In experiment $1 m C r y 1^{-/}$and $m C r y 2^{-/-}$mice as well as $m \operatorname{Per} 1^{B r d m 1}$ and $m P e r 2^{B r d m 1}$ mutant mice were exposed to 15 minute light pulses during the first cycle following entrainment, either early (ExT20) or late (ExT4) in the subjective night. In experiment 2 a full PRC was measured for all these strains by exposure to light pulses of the same duration and intensity in freerunning conditions in constant darkness. Directly after entrainment (experiment 1 ), $m$ Per $1^{\text {Brdm1 }}$ animals did not show significant phase advances by a light pulse in the late subjective night (ExT4), as in the study by Albrecht et al. (2001). In the same experiment $m P e r 2^{B r d m 1}$ mice became arrhythmic too frequently to reliably measure their phase responses. Mice with a targeted gene disruption in $m C r y 1$ or $m C r y 2$ showed increased phase delays compared to wildtype after exposure to a light pulse in the early subjective night (ExT20). Otherwise, phase shifts were not significantly affected. In freerun (experiment 2), all genotypes did show phase advances and phase delays. The $m$ Per2 ${ }^{\text {Brdm1 }}$ mutant PRC was above the mPer $1^{\text {Brdm1 }}$ mutant and wildtype PRC (i.e., less delayed and more advanced) at most circadian phases. The $m P e r 1^{\text {Brdm1 }}$ mutant PRC was not distinguishable from the wildtype PRC. $m C r y 2^{--}$mice showed much smaller phase delays than $m C r y 1^{--}$mice in the subjective evening (delay phase). In general, $m$ Per $2^{\text {Brdm1 }}$ mutant mice were more accelerated by light compared to $m P e r 1^{\text {Brdm1 }}$ and wildtype control mice, whereas $m \mathrm{Cry}^{-1^{-}}$mice were more delayed by light than $m \mathrm{Cry} 2^{-{ }^{-}}$mice.

Key words circadian clock, phase resetting, Cry1, Cry2, Per1, Per2, PRC 


\section{Introduction}

The notion that the circadian pacemaker in nocturnal rodents is built upon two components with opposite responses to light has a long history (Pittendrigh and Daan, 1976a; Ilnerová and Vanecek, 1982; Wehr, 1997). This hypothesis gained new interest when Jagota et al. (2000) demonstrated that at the physiological level there are indeed two subsystems in the SCN that respond differentially to changing daylength. Together with the dual nature of the genetic make-up of the molecular mechanism these findings led Daan et al. (2001) to postulate two genetic components in the core mechanism. They derived a series of predictions on the behavior in rodent systems where one or the other component has been genetically deleted. According to the hypothesis, Cry1 and Per1 are involved in a component of the system that is accelerated by light, and decelerated in darkness, where the Per2 and Cry2 gene are involved in a component that is decelerated by light and accelerated by darkness. These properties were expected to be phenotypically reflected in freerun in DD, in constant light, and in phase responses of the circadian system by light pulses in mice with a functional deletion in either of these genes.

The hypothesis was partly inspired by and based on a study by Albrecht et al. (2001) who demonstrated absence of phase advances in mPer1 mutant mice and absence of phase delays and increased phase advances in mPer 2 mutant mice after exposure to light pulses early (Zeitgeber Time ZT 14) and late (ZT 22) in the subjective night, respectively. These light pulses were applied just after entrainment in a so-called type II protocol (Aschoff, 1965). In such a protocol the phase prior to the light pulse is derived from the rhythm under LD conditions and hence subject to masking influences, while there is no masking in the freerun after the light pulse. This may affect the measurement of the phase shift. In addition, different circadian genotypes may have systematically deviating phase angle differences with the LD cycle. It is therefore desirable to evaluate the phase 
shifts relative to an unpulsed control experiment, and obtain a precise quantitative evaluation of the shift. In this study, this protocol has been applied (in experiment 1) to $m \mathrm{Cry}^{-/-}$and $m \mathrm{Cry}^{-{ }^{--}}$mice and simultaneously, in a repeat of Albrecht et al.'s (2001) work, to $m P e r 1^{\text {Brdm1 }}$ and $m P e r 2^{\text {Brdm1 }}$ mice.

Suppression by a genetic disturbance of the induction of either phase delays or phase advances by light pulses given at two time points in the cycle does not prove that all phase delays or advances are suppressed. Such suppression might in fact also be interpreted as resulting from a shift in the PRC relative to the zeitgeber as expected if the circadian period is affected. Therefore, we obtained in addition a full PRC in a freerunning situation under constant darkness (DD), a type I protocol (Aschoff, 1965). This was done in experiment 2 in all genotypes (except wildtype with the same background as mCry1-/- and mCry2-/-) with light pulses of the same duration and intensity as in experiment 1 . The results prove that all four genotypes retain the capacity for both advancing and delaying responses to light pulses, while at the same time revealing interesting differences between the genotypes in resetting behavior. 


\section{Methods}

The $m C r y 1^{-/}, m C r y 2^{-/}, m P e r 1^{B r d m 1}$ and $m P e r 2^{B r d m 1}$ mice have been described previously (van der Horst et al., 1999; Zheng et al., 1999). They were generated in a hybrid C57BL/6 x 129ola ( $m$ Cry mice) and C57BL/ 6 x 129 SvEvBrd genetic background (mPer mice).

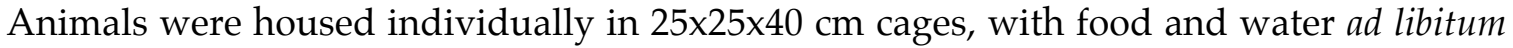
in a sound-attenuated and climatized room with 90 cages. Spontaneous locomotor activity was recorded with running wheels $(\varnothing 14 \mathrm{~cm})$ connected to an Event Recording System (ERS) storing wheel revolutions in 2 minute intervals. Temperature was maintained at $23 \pm 1{ }^{\circ} \mathrm{C}$ throughout the two experiments.

\section{Experiment 1: Phase responses in entrainment.}

90 Mice, all males (age 1 - 2 months), were used in the study: 15 homozygous $m$ Cry1 $\left(m \mathrm{Cry}^{-/-}\right)$mutant mice, 15 homozygous $m \mathrm{Cry} 2$ mutant mice $\left(m \mathrm{Cr} y 2^{-/}\right.$; van-der-Horst et al., 1999), 15 wildtype mice with the same background as the $\mathrm{mCry}$ mutant mice, $15 \mathrm{mPer}$ mutant mice $\left(m P e r 1^{\text {Brdm1 } 1}\right), 15$ mPer2 mutant mice $\left(m P e r 2^{\text {Brdm1 }}\right.$; Zheng et al., 1999) and 15 wildtype mice with the same background as the mPer mutant mice. The experiment was designed to repeat the study by Albrecht et al. (2001) on phase shifts in response to light pulses immediately following entrainment by an LD 12:12 cycle in the $\mathrm{mPer}^{\mathrm{Brdm} 1}$ mutant mice, while simultaneously expanding the analysis to the $m C r y$ gene targeted mutant mice.

All mice were entrained for 14 days in LD 12:12. They were then released into constant darkness (DD) except for a light pulse presented in the first cycle during the subjective night at External Time ExT20 (which in LD 12:12 equals Zeitgeber Time ZT14, see Daan et al., 2002). This was followed by 14 days of freerun in DD. The procedure was repeated once with a light pulse at ExT4 (ZT22) and once without a light pulse. This last treatment was done in order to assess the initial phase in a control situation after the transition 
from LD to DD. All light pulses had a duration of $15^{\prime}$ and intensity of 480 Lux (700 $\mathrm{mW} / \mathrm{m}^{2}$ at the cage floor level; white fluorescent tube light 36W/85). All cages had equal distance to their light source, light pulse intensity was checked for all cages to deviate no more than $\pm 140 \mathrm{Lux}\left(200 \mathrm{~mW} / \mathrm{m}^{2}\right)$ at the cage floor level.

\section{Experiment 2: Phase responses in freerun}

75 Mice, all males (age $1-2$ months), were used in the study: 15 homozygous $m C r y 1^{--}$ mice, 15 homozygous $m C r y 2^{-/}$mice, 15 homozygous $m P e r 1^{B r d m 1}$ mice, 15 homozygous $m P e r 2^{B r d m 1}$ mice and 15 wildtype mice with the same background as the mPer mutant mice. At the time we did this experiment, we had insufficient wildtype mice with the appropriate background for the $m C r y$ mutant mice available, and therefore $m C r y^{-/}$mice results can only be compared between the $m \mathrm{Cry}^{-1_{-}^{-}}$and $m \mathrm{Cry}^{2-{ }_{-}^{--}}$strains. All mice were entrained for 14 days in LD 12:12 and then left in constant darkness (DD) for 176 days, interrupted only by brief light pulses with equal duration (15') and intensity (480 Lux) as in experiment 1, followed by 12 days of freerun. Due to the slight intraindividual variations in circadian period length within each genotype and subsequent differences in phase shifts by consecutive light pulses, the light pulses eventually became spread over the entire circadian cycle allowing the construction of a full phase response curve for each of the five genotypes. All $m P e r 1^{B r d m 1}, m C r y 1^{--}$and $m C r y 2^{--}$mice were exposed to 11 light pulses. $m P e r 2^{\text {Brdm1 }}$ mice in our lab often become arrhythmic in constant darkness, although rhythmicity may recur spontaneously following a light pulse. Thereby this strain initially yielded insufficient data for the reconstruction of a complete PRC. The experiment was therefore extended with another batch of $30 \mathrm{mPer} \mathrm{B}^{\mathrm{Brdm} 1}$ mice that were exposed to 4 additional light pulses. 


\section{Analysis}

For both experiments, phase shifts were calculated by applying a new quantitative computation method. This method uses custom designed software, as specified in detail in the appendix. Briefly, what the software package does is calculate the phase of the circadian activity rhythm (in Internal Time InT) at exactly the time of the light pulse on the basis of forward extrapolation from the rhythm during cycles -10 till -1 (before the light pulse). Then it calculates the phase at that time again on the basis of backward extrapolation from the rhythm during cycles 3 till 12 (after the light pulse). The difference between the two computed phases at exactly the same time at the onset of the light pulse is the actual phase shift. It is given a positive sign (advance) when the second phase calculation yields a smaller phase angle than the first. It is given a negative sign (delay) when the second phase calculation yields a larger phase angle than the first. Activity data from the first 48 hours after each light pulse were omitted to avoid potential transient $\tau$ values evoked by the light pulse.

The two phase angles are calculated by first determining the period before $\left(\tau_{1}\right)$ and after $\left(\tau_{2}\right)$ the light pulse through periodogram analysis, and by defining different phase markers on the wave form of the time series stacked with these periods. Activity onset was the most precise phase marker as is usual in running wheel data (see Daan and Oklejewicz, 2003) and was used for phase definition. Activity onset was defined as InT (Internal Time) 18. 


\section{Results}

\section{Experiment 1}

The first experiment was designed to establish the phase shift induced by a $15^{\prime}$ light pulse at two different phases in the first cycle following entrainment to an LD 12:12 cycle. The protocol was an exact copy of the protocol used by Albrecht et al. (2001) and is known as a type II protocol (Aschoff, 1965; Mrosovsky, 1996). The phase at the time of the light pulse is assessed by extrapolation from the (entrained) rhythm before the light pulse and back-extrapolation from the (freerunning) rhythm after the pulse.

The difficulty with this protocol is that the activity onset under entrainment is affected by masking, and thereby tends to occur at a later circadian phase than the activity onset in the subsequent freerunning situation (see Figure 1). This is

[position Figure 1 about here; Width = one column; Height $=2-2.5$ inches]

potentially different between genotypes, since mutations of circadian genes tend to elicit different freerunning periods and hence different phase angles in entrainment (Figure 2). To accommodate this difference we also analyzed a release into freerun without a preceding light pulse, as was done also by Albrecht et al (2001).

\section{[position Figure 2 about here; Width $=$ one column; Height $=\sim 2$ inches]}

The phase shifts obtained in this protocol were thus defined as the intraindividual differences in phase during freerun following the light pulse and the phase without a light pulse. This procedure did lead to loss of data when animals in the latter control situation became arrhythmic - as often happened in $m P e r 2^{\text {Brdm1 }}$ (Oster et al., 2002) - and no phase could be established. Examples of behavioral shifts are presented in actograms in figure 3; the resulting average phase shifts for the $m$ Cry genotypes are plotted in figure 4 .

[position Figure 3 about here; Width = two columns; Height $=\sim 3.5$ inches] 


\section{[position Figure 4 about here; Width $=$ one column; Height $=\sim 6$ inches]}

In response to the light pulse at ExT20 $m P e r 1^{B r d m 1}$ mice did show a small average delay phase shift of $-1.2 \mathrm{~h}$ (s.e.m. $1.1 \mathrm{~h}$ ). The advance phase shift following a light pulse at ExT 4 was on average 0.5 h (s.e.m. 0.3 h). These shifts were not significantly different from zero. They are therefore not in disagreement with the data presented by Albrecht et al (2001). Since our analysis required individual mice to have clear rhythmic behavior both with and without a light pulse, we could not collect sufficient data for $m P e r 2^{\text {Brdm1 }}$ mice in experiment 1 due to its frequent arrhythmicity.

When released in DD after entrainment, both $m \mathrm{Cry}^{--}$and $m \mathrm{Cry}^{-{ }^{--}}$mice did show a considerable average phase advance without light pulses. The average advances in both $m \mathrm{Cry}^{-/-}$and $m \mathrm{Cry}^{-/-}$mice were larger than in wildtype (One way ANOVA on ranks; $\mathrm{H}=12.0 ; \mathrm{df}=2 ; p<0.005)$. After exposure to a light pulse at ExT20, wildtype mice exhibited a normal and significant delay. Both $m C r y 1^{--}$and $m C r y 2^{---}$mice did show considerable delays after this light pulse, larger than wildtype mice (One way ANOVA, F=8.3; DF=2; $p=0.001)$ with both $m C r y 1^{-/-}$and $m C r y 2^{-/-}$significantly different from wildtype (Tukey Test, $p<0.05 ; p<0.05)$. Delays in $m \mathrm{Cry1}^{-/-}$and $m \mathrm{Cr} y 2^{--}$mice were statistically not distinguishable from each other. When exposed to a light pulse at ExT4, $m \mathrm{Cry}^{-/}$and $m \mathrm{Cry}^{-/-}$mice both showed small average phase advances similar to and not significantly different from those in wildtype mice.

\section{Experiment 2}

In total 139 phase shifts could be measured in DD in wildtype mice (background similar to that of the $m P e r$ mutants), 79 in $m P e r 1^{\text {Brdm1 }}, 71$ in $m P e r 2^{\text {Brdm1 }}, 86$ in $m C r y 1^{-1}$ and 98 in $m \mathrm{Cry}^{-/-}$animals. Examples of phase shifts in all genotypes are presented in figure 5. Phase shifts were excluded if (1) the peak $\Delta \mathrm{Qp}$ value obtained by 
[position Figure 5 about here; Width $=$ two columns; Height $=\sim 3.5$ inches]

periodogram analysis (Sokolove and Bushell, 1978; $\Delta \mathrm{Qp}$ is the value for $\mathrm{Q}$ at the peak minus the corresponding $0.1 \%$ significance value) was smaller than zero for the circadian activity rhythms either before or after a light pulse, and (2) if one of the onsets before or after the light pulse were fitted in obvious disagreement with visual inspection of the actogram. This occurred for instance when rhythms were ultradian and the positive $\Delta \mathrm{Qp}$ value was due to a sub harmonic. It could also be caused by obvious pattern changes. Criterion (2) excluded 19 shifts in $m P e r 1^{\text {Brdm1 }}, 22$ shifts in $m P e r 2^{\text {Brdm1 }}, 9$ shifts in wildtype, 17 shifts in $m C r y 1^{--}$and 15 shifts in $m C r y 2^{--}$mice.

\section{$\underline{\text { Rhythmicity in DD }}$}

$m P e r 2^{B r d m 1}$ yielded the smallest number of phase shifts, in spite of the extension of the study for this strain. This is again due to the fact that these mice often become arrhythmic in DD (Zheng et al. 1999; Oster et al 2002). Arrhythmicity often started after a light pulse and could also suddenly disappear after another light pulse (fig. 5, right $m P e r 2^{\text {Brdm1 }}$ actograms). We analyzed whether the distribution (see fig. 6A) over

\section{[position Figure 6 about here; Width $=$ two columns; Height $=\sim 3$ inches]}

the circadian cycle of light pulses that were followed by arrhythmicity differed from random. Responses (binary data) were transformed by the logit link function and analyzed assuming a binomial error distribution on the individual level. We tested in MLwin 1.1 (Bryk and Raudenbusch, 1993) in a two level model using individual responses whether there was heterogeneity with respect to phase. Second order penalized quasilikelihood estimation was used (Goldstein, 1995); there was no bias towards a particular phase for light pulses followed by arrhythmicity (Walt statistic $\chi^{2}$ test; $p=0.32)$. 
In addition we analyzed whether light pulses presented to arrhythmic $m P e r 2^{B r d m 1}$ mice that became rhythmic immediately after a light pulse $(n=96)$ were concentrated around a particular phase of the new rhythm. Figure $6 \mathrm{~B}$ demonstrates that these new phases concentrate around InT 13.8. This concentration is decidedly different from a random distribution $\left(\chi^{2}\right.$ test; $\left.p<0.001\right)$. Hence the pacemaker apparently spontaneously can resume self-sustained DD motion after a light pulse around this circadian phase.

In freerun in DD, circadian period lengths measured in between the light pulses were on average $23.5 \pm 0.2 \mathrm{~h}$ in $m \operatorname{Per} 1^{B r d m 1}$ mice, i.e., not significantly different from wildtype $(23.5 \pm 0.1 \mathrm{~h}) ; 22.7 \pm 0.1 \mathrm{~h}$ in $m P e r 2^{B r d m 1}$ mice (significantly shorter than in wildtype, $\mathrm{t}$-test, $p<0.001)$. The average period was $21.7 \pm 0.1 \mathrm{~h}$ in $m \mathrm{Cry}^{-/-}$mice, significantly shorter than in $m \mathrm{Cry}^{-/-}$mice $(25.1 \pm 0.1 \mathrm{~h}$, t-test, $p<0.001)$, in agreement with the results of van der Horst et al. (1999).

\section{$\underline{\text { Phase response curves }}$}

To obtain a quantitative comparison of the PRC's among the genotypes, we first combined data in 12 bins of 2 circadian hours width, and calculated the average phase shift per 2-h phase bin for each strain. These data are plotted, along with the standard errors of the means, in figure 7. The first conclusion from this figure is that all genotypes apparently are capable of both phase advances and phase delays,

\section{[position Figure 7 about here; Width $=$ one columns; Height $=\sim 6$ inches]}

although the amplitude of the advance section of the PRC's tends to be smaller than that of the delay section. The wildtype PRC corresponds reasonably well with the PRC published for 15' light pulses in C57Bl6 mice by Daan \& Pittendrigh (1976). In the quantitative detail there are differences. 
The PRC for $m P e r 2^{\text {Brdm1 }}$ appears to show larger advances and smaller delays than the $m P e r 1^{B r d m 1}$ mutant and wildtype mice, while delays in $m C r y 2^{-\nu_{-}}$on average are smaller than in $m \mathrm{Cry}^{-/-}$mutant mice. We tested the general differences first in ANOVA's on the basis of all 12 bins (table 1) and then in 3 groups of 4 bins, roughly representing the Delay zone (InT 18-02), the Advance zone (InT 02-10) and the Dead zone (InT 10-18).

In a two-way ANOVA test, the overallmPer2 ${ }^{\text {Brdm1 }}$ PRC is significantly above the $m P e r 1^{B r d m 1}$ PRC and the wildtype PRC, and the $m C r y^{-/}$PRC is significantly below the $m C r y 2^{-/-}$PRC. When the PRC's are tested in three sections, a delay section (InT18 - InT2), an advance section (InT2 - InT10) and in the dead zone section (InT10 - InT18), significant differences remain present between $m P e r 2^{B r d m 1}$ and wildtype mice in the advance and delay section. The average delay in $m \mathrm{Cry}^{-/-}$was highly significant above the average in $m \mathrm{Cry}^{-/}$mice (table 1). Significant differences in other sections between different genotypes within strains are absent.

[position Table 1 about here; Width = two columns; Height $=\sim 3$ inches] 


\section{Discussion}

mPer mutants

The results for light pulses presented to $m \operatorname{Per} 1^{\text {Brdm1 }}$ immediately after entrainment in either the early part or the late part of the subjective night (experiment 1) are not different from the results reported by Albrecht et al. (2001) carried out in the same type II protocol (Aschoff 1965). Our data for $m P e r 2^{\text {Brdm1 }}$ mice turned out to be far too few as a consequence of the frequent arrhythmicity in this strain in DD (Zheng et al 1999; Oster et al 2002), that often occurred either in the pulse or in the control situation. Thus, we can neither refute nor firmly confirm the observations by Albrecht et al. (2001) of suppressed delay responses to light in mPer 2 mutant mice.

The full PRC's of $m P e r 1^{\text {Brdm1 }}$ and $m P e r 2^{\text {Brdm1 }}$ show that both genotypes indeed can exhibit both phase advances and phase delays. The $m \operatorname{Per} 1^{\text {Brdm1 }}$ PRC is in fact virtually on top of the wildtype PRC in the phase delay area, and there are no significant differences between these two strains anywhere in the cycle. The difference in response to an ExT4 light pulse in the data of Albrecht et al (2001) is not reflected in the full PRC's. This difference is possibly due to different protocols. Differences between LD and DD in the phase relationship between gene expression patterns and locomotor activity, and also between individual neurons in the SCN have been demonstrated by Quintero et al. (2003). A difference in phase relationship might still be present in vivo in the first cycles after transition from $\mathrm{LD}$ to $\mathrm{DD}$, where light pulses in the type II protocol are administered.

Statistical comparison (Table 1) shows that the PRC's of the two mPer genotypes differ in the direction of the observations by Albrecht et al. (2001), with more advances in $m P e r 2^{B r d m 1}$ mice. The differences in the amplitude of phase shifts from the data from Albrecht et al. (2001) may be attributable to the two different protocols, even if such protocol differences between Aschoff's type I and type II protocols have not been 
observed for nonphotic stimuli to hamsters (Mrosovsky, 1996). A difference between responses in the two protocols may be evoked by the light exposure during entrainment in the type II protocol that may reduce the amplitude of the phase shift. It has long been known that entrainment increases the amplitude of the oscillator ('resonance') and simultaneously reduces the amplitude of the phase shift in response to standard pulses, such that there is slow dark adaptation (Winfree, 1972; Refinetti, 2003).

It is of importance to note that the $m P e r 2^{B r d m 1}$ strain, although showing smaller delay shifts, is surely not insensitive to light. On the contrary, the mPer2 mutants frequently responded to light pulses with immediate complete arrhythmia, and this effect did not depend on the circadian phase exposed to the light. On the other hand, brief light pulses given to arrhythmic animals were often followed by a spontaneous return of rhythmicity. This is reminiscent of the induction of rhythmicity by longer light pulses described in clock mutant mice (Vitaterna et al., 1994; Spoelstra et al., 2002). Apparently, the homozygous clock and per2 mutant share lability of the circadian system in DD and

the stabilizing response to single brief light exposures. In $m P e r 2^{B r d m 1}$ the returning circadian rhythm started from around circadian phase InT 13.8 in the majority of cases. In the clock mutant this has not been investigated.

\section{mCry mutants}

The phase delays observed in $m C r y 1^{-/}$and $m \mathrm{Cry}^{-/-}$after entrainment (experiment 1 ) were significantly larger than in wildtype. This is possibly related to the fact that without a light pulse both showed a significantly earlier phase than wildtype (Figure 4). Thereby the light pulse aimed at ExT20 may have hit the circadian system at a slightly later phase than in wildtype. The difference attests to the difficulty in using the type II protocol for PRC studies. The small phase advances following light pulses at ExT4 were not affected by the phase difference, possibly because around this phase there are less 
steep changes in the PRC. The genotypic differences in PRC's are more completely reflected in freerun (experiment 2). There were particularly distinct differences in the phase delays produced in $m$ Cry mutant mice in the delay phase between InT 18 and InT 02. This was fully due to strong and highly significant suppression of the phase delay shifts in $m \mathrm{Cry}^{-/-}$, at least when compared to $m \mathrm{Cry}^{-{ }^{-}}$(table 1 ).

\section{Conclusion}

Taken together, phase responses are more positive and/or less negative in mPer2- than in mPer1- mutants, and the same is true of $m C r y 2$ - compared to $m C r y 1$-mutants. This means that mPer2- and $m C r y 2-$ mutant circadian systems are more accelerated and/or less decelerated by light, than mPer1- and mCry1- mutants, respectively. At cursory inspection, these results seem to qualitatively fit with the predictions generated from the molecular 2-component model for rodent circadian systems (Daan et al., 2001). However, that model made more specific predictions concerning the difference from wildtype. A difference from wildtype has so far been found only in the mPer2 mutant. This mutant has dominant other characteristics, such as the tendency to become arrhythmic in DD, which may be related to the deviant PRC. Therefore we have to be careful in interpreting the results as supporting the 2-component hypothesis. More work is needed employing different protocols to arrive at a firm conclusion in this respect. Be this as it may, the data demonstrate clearly that all mutant strains retain both advancing and delaying responses. All can be expected to entrain to both longer and shorter T's, as observed by Bae and Weaver (2003) for Per mutants although the ranges of entrainment may show only partial overlap.

\section{Acknowledgments}


This study was supported by BrainTime (EC $5^{\text {th }}$ framework grant QLRT-2001-01829). We thank G. J. F. Overkamp for expert technical support, and three anonymous reviewers for their help in substantially improve the manuscript. 


\section{REFERENCES}

Albrecht U, Zheng B, Larkin DW, Sun ZS, and Lee CC (2001) mPerl and mPer2 Are Essential for Normal Resetting of the Circadian Clock. Journal of Biological Rhythms 16:100-104.

Aschoff J (1965) Response Curves in Circadian Periodicity, in Circadian Clocks, Aschoff J, eds, pp 95-111, North Holland Publishing Company, Amsterdam

Bae K and Weaver DR (2003) Light-Induced Phase Shifts in Mice Lacking mPER1 or mPER2. J.Biol.Rhythms 18:123-133.

Bryk AS and Raudenbusch SW (1993) Hierarchical Linear Models: Application and Data Analysis Method. Sage, Newbury Park.

Daan S, Albrecht U, van-der-Horst GTJ, Roenneberg T, Roenneberg T, Wehr TA, and Schwarz WJ (2001) Assembling a Clock for All Seasons: Are There M and E Oscillators in the Genes? Journal of Biological Rhythms 16:105-116.

Daan S, Merrow M, and Roenneberg T (2002) External Time - Internal Time. J.Biol.Rhythms 17:107-109.

Daan S and Oklejewicz M (2003) The Precision of Circadian Clocks: Assessment and Analysis in Syrian Hamsters. Chronobiol.Int. 20:209-221.

Daan S and Pittendrigh CS (1976) A Functional Analysis of Circadian Pacemakers in Nocturnal Rodents II. The Variability of Phase Response Curves. J.comp.Physiol. 106:253-266.

Goldstein H (1995) Multilevel Statistical Models. Edward Arnold, London.

Ilnerová H and Vanecek J (1982) Two-oscillator structure of the pacemaker controlling the circadian rhythm of $\mathrm{N}$-acetyltransferase in rat pineal gland. J.comp.Physiol [A]. 145:539-548.

Jagota A, de la Iglesia HO, and Schwarz WJ (2000) Morning and evening circadian oscillations in the suprachiasmatic nucleus in vitro. Nature neuroscience 3:372376. 
Mrosovsky N (1996) Methods of measuring phase shifts: why I continue to use an Aschoff type II procedure despite the skepticism of referees. Chronobiol.Int. 13:387-392.

Oster H, Yasui A, van-der-Horst GTJ, and Albrecht U (2002) Disruption of $m C r y 2$ restores circadian rhythmicity in $m P e r 2$ mutant mice. GENES \& DEVELOPMENT 16:2633-2638.

Pittendrigh CS and Daan S (1976a) A Funcional Analysis of Circadian Pacemakers in Nocturnal Rodents V. Pacemaker Structure: A Clock for All Seasons. J.comp.Physiol. 106:333-355.

Quintero JE, Kuhlman SJ, and McMahon DG (2003) The Biological Clock Nucleus: A Multiphasic Oscillator Network Regulated by Light. J.Neuroscience 23:80708076.

Refinetti R (2003) Effects of Prolonged Exposure of Darkness on Circadian Photic Responsiveness in the Mouse. Chronobiol.Int. 20:417-440.

Sokolove PG and Bushell WN (1978) The Chi Square Periodogram: Its Utility for Analysis of Circaidan Rhythms. J.theor.Biol. 72:131-160.

Spoelstra K, Oklejewicz M, and Daan S (2002) Restoration of Self-Sustained Circadian Rhythmicity by the Mutant Clock Allele in Mice in Constant Illumnation. Journal of Biological Rhythms 17:520-525.

van der Horst GTJ, Muijtjens M, Kobayashi K, Takano R, Kanno S, Takao M, de Wit J, Verkerk A, Eker APM, van Leenen D, Buijs R, Bootsma D, Hoeijmakers JHJ, and Yasui A (1999) Mammalian Cry1 and Cry2 are essential for maintenance of circadian rhythms. Nature 398:627-630.

Vitaterna MH, King DP, Chang A-M, Kornhauser JM, Lowrey PL, McDonald JD, Dove WF, Pinto LH, Turek FW, and Takahashi JS (1994) Mutagenesis and Mapping of a Mouse Gene, Clock, Essential for Circadian Behavior. Science 264:719-725.

Wehr TA (1997) Melatonin and seasonal rhythms. J.Biol.Rhythms 12:517-526.

Winfree AT (1972) Slow Dark-Adaptation in Drosophila's Circadian Clock. J.comp.Physiol. 77:418-434. 
Zheng B, Larkin DW, Albrecht U, Sun ZS, Sage M, Eichele G, Lee CC, and Bradley A (1999) The $m P e r 2$ gene encodes a functional component of the mammalian circadian clock. Nature 400:169-173. 


\section{Appendix}

Assessing phase shifts from automatically collected data in a standardized, automated way has always been a complicated issue. To calculate accurate phase shifts in a standardized way, proper phase markers are of utmost importance. Usually, phase markers are detected for each individual cycle in an entrained or freerunning rhythm. In freerun, the circadian system reverts to its original steady state after the light pulse. Phase shifts are assessed by drawing lines (regression or eye fitted) trough consecutive phase markers for freeruns before and after a light pulse. Although inter-observer consistency in eye fitting procedures (Pittendrigh and Daan, 1976b) has often been considered to support the precision of the procedure, this does not mean that the procedure is also accurate. To obtain an objective measure of phase shift, we developed an automatic means of phase calculation where phase markers before and after a light pulse are based on all circadian cycles. The software that performs these calculations will be made available by the first author on request. The calculations proceed in 4 steps:

Step 1. The program first calculates the period length of the activity rhythm before $\left(_{-1}\right)$

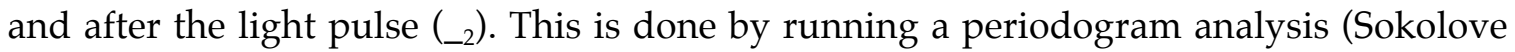
and Bushell, 1978) on all activity data starting from 10 days before the light pulse till the time the light pulse starts, and again on all activity data from 2 days till 12 days after the light pulse. Data from the first 2 days after the light pulse are omitted to exclude transient cycles. All calculations described are based on these two time windows.

Step 2. The circadian activity pattern before and after the light pulse is averaged over $\_1$ and $\_2$, respectively. This is done by averaging the activity values with a distance equal to a multiple of _ in _/(sample interval) tallies, the first tally starting with the first sample of the data range (see figure 8).

[position Figure 8 about here; Width $=$ one column; Height $=\sim 4.5$ inches] 
Step 3. The program calculates the (circular) center of gravity in each of the two average activity patterns. It then searches the first time interval where the activity count exceeds the average value of all tallies, starting $0.5^{*}$ - prior to the center point of gravity. The offset of activity is detected in the same way, but then by going backwards in time starting $0.5^{*}$ after the center point of gravity. The program can be instructed to carry out this procedure on either the raw data or on running means over adjustable time windows. In the analyses in this article we always used 1-h running means.

Step 4. The detected phase markers are projected on the actogram (see figure 8 ). The straight lines denote onsets, the medium dashed lines denote the center points of gravity, the dashed lines denote the offsets of activity. The actual phase shift is defined as the difference between the phase angle at the time of the light pulse calculated on the basis of the onset of activity before $\left(_{-1}\right)$ and after the light pulse $\left(_{2}\right)$. The Internal Time of ${ }_{-1}$ and $\_$are calculated as follows:

$$
\begin{aligned}
& \varphi_{1}=\left(\left(\left(t-\text { ons }_{1}\right) \bmod \tau_{1}\right) * 24 / \tau_{1}+18\right) \bmod 24 \operatorname{hours}(\operatorname{In} T) \\
& \varphi_{2}=\left(\left(\left(t-\text { ons }_{2}\right) \bmod \tau_{2}\right) * 24 / \tau_{2}+18\right) \bmod 24 \operatorname{hours}(\operatorname{In} T)
\end{aligned}
$$

Where $t L P=$ time of exposure to the Light pulse;

ons $_{1}=$ moment of onset derived from the average activity pattern before the light pulse;

ons $_{2}=$ moment of onset derived from the average activity pattern after the light pulse.

With these two phase angles of the light pulse the actual phase shift is calculated:

$\Delta \varphi=\varphi_{2}-\varphi_{1}$ (Circadian hours) 


\section{Figure legends}

Fig. 1. Application of an advancing light pulse in the subjective morning in a type-II protocol (Aschoff 1965). __ measured from free running activity onset

relative to prior activity onset $\left(\_\right)$and relative to prior lights off $\left(\_\right.$' $)$yields different values when mice start activity before lights off.

Fig. 2. Average position of the onset of activity relative to lights off $(t=0)$ during entrainment in LD 12:12 in different genotypes. Error bars indicate 1 s.e.m.

Fig. 3. Examples of phase shifts after transition from L:D 12:12 to DD (upper row); with an additional 15' 480 Lux light pulse at ExT20 (middle row) and at ExT4 (bottom row) in different genotypes. Gray indicates darkness; light pulses are denoted by open squares.

Fig. 4. Average phase shifts \pm 1 s.e.m. directly after entrainment in $m \mathrm{Cry}$ mutant mice. Phase shifts are calculated based on activity onset and intraindividually corrected for the spontaneous phase shift after transition from L:D 12:12 to DD. Upper graph: no light pulse given; Middle graph: light pulse starting $2 \mathrm{~h}$ after last lights-off (ExT 20); Bottom graph: light pulse starting $10 \mathrm{~h}$ after last lights-off (ExT 4). Significant differences from wild type are denoted by asterisks. 
Fig. 5. Examples of phase delays (upper row) and phase advances (lower row) in running wheel behavior in freerun in DD in mutant mice, as result of a $15^{\prime} 480$ Lux light pulse (indicated by open squares). Left $m$ Per $2^{\text {Brdm1 }}$ actograms: arrhythmicity after exposure to a light pulse (upper actogram) and rhythmicity after exposure to a light pulse in a formerly arrhythmic mouse. Activity patterns are plotted at intrinsic_ basis, denoted below each actogram.

Fig. 6. A. Frequency distribution of circadian phases of light pulses in $m P e r 2^{\text {Bmdr1 }}$ mice that became arrhythmic directly after light exposure. B. Frequency distribution of circadian phases calculated backwards for the time of the light pulse in $m P e r 2^{B r d m 1}$ mice that were arrhythmic before and regained rhythmicity directly after light exposure.

Fig. 7. Phase response curves of different strains of mice binned in $2 \mathrm{~h}$ intervals. Each symbol indicates the mean phase shift of all circadian rhythms in a strain illuminated by a light pulse starting in a two-hour interval of circadian phase (Internal Time, defined by InT 18 = activity onset). Lines indicate 1 s.e.m. on both sides of the mean; numbers above the graphs denote the $\mathrm{n}$ size for each genotype for each bin.

Fig. 8. The automatic phase shift calculation method. Actogram of running wheel activity data plotted on basis of the average of _ before $\left(_{\_} 1\right)$ and _ after $\left(\__{2}\right)$ the light pulse. Data omitted to avoid effects of transient tau values are plotted in grey. 
The open circle indicates the light pulse. Upper and lower graph: average activity pattern before and after the light pulse, respectively. Straight lines denote onset of activity, medium dashed lines denote circular center of gravity, dashed lines denote offset of activity. The grey arrow indicates the actual phase shift. 


\begin{tabular}{|c|c|c|c|c|c|c|c|c|c|c|}
\hline \multicolumn{2}{|c|}{ Circadian Phase } & \multicolumn{2}{|c|}{$\ln T 0-24$} & \multicolumn{2}{|c|}{$\ln T 18$ - $02(D)$} & \multicolumn{2}{|c|}{$\operatorname{InT} 02-10(\mathrm{~A})$} & \multicolumn{2}{|c|}{$\ln T 10-18$} & \multirow[b]{2}{*}{$D / A$} \\
\hline & & mean & (SEM; n) & mean & $(S E M ; n)$ & mean & (SEM; $n)$ & mean & $(S E M ; n)$ & \\
\hline & WT (Per) & -0.49 & $(0.13 ; 139)$ & -1.90 & $(0.17 ; 43)$ & 0.31 & $(0.18 ; 58)$ & -0.06 & $(0.12 ; 38)$ & 6.13 \\
\hline & $m P e r 1^{\text {Brdm1 }}$ & -0.16 & $(0.18 ; 79)$ & -1.30 & $(0.34 ; 24)$ & 0.53 & $(0.22 ; 31)$ & 0.10 & $(0.28 ; 24)$ & 2.47 \\
\hline & $m P e r 2^{\text {Brdm1 }}$ & 0.09 & $(0.23 ; 71)$ & -1.32 & $(0.32 ; 27)$ & 1.43 & $(0.31 ; 23)$ & 0.44 & $(0.21 ; 21)$ & 0.92 \\
\hline \multirow[t]{3}{*}{ ANOVA } & $\mathrm{F}$ & 5.0 & & 4.2 & & 4.1 & & 0.5 & & \\
\hline & df & 2 & & 2 & & 2 & & 2 & & \\
\hline & $p$ & $<0.01$ & & $<0.05$ & & $<0.05$ & & ns & & \\
\hline \multicolumn{11}{|l|}{ Tukey } \\
\hline$m P e r 1^{\text {Brdm1 }}$ & WT (Per) & ns & & ns & & ns & & ns & & \\
\hline mPer2 ${ }^{\text {Brdm1 }}$ & WT (Per) & $<0.01$ & & $<0.05$ & & $<0.05$ & & ns & & \\
\hline \multirow[t]{3}{*}{$m P e r 1^{\text {Brdm1 }}$} & $m P e r 2^{\text {Brdm1 }}$ & $<0.05$ & & ns & & ns & & ns & & \\
\hline & mCry $1^{-/}$ & -0.86 & $(0.18 ; 86)$ & -2.45 & $(0.20 ; 34)$ & 0.35 & $(0.24 ; 29)$ & -0.041 & $(0.14 ; 23)$ & 7.00 \\
\hline & $m C r y 2^{-/}$ & -0.13 & $(0.11 ; 98)$ & -0.95 & $(0.19 ; 32)$ & 0.78 & $(0.13 ; 30)$ & -0.145 & $(0.09 ; 36)$ & 1.23 \\
\hline \multirow[t]{3}{*}{ ANOVA } & $\mathrm{F}$ & 16.5 & & 29.5 & & 2.0 & & 1.6 & & \\
\hline & df & 1 & & 1 & & 1 & & 1 & & \\
\hline & $p$ & 0.001 & & $<0.001$ & & ns & & ns & & \\
\hline
\end{tabular}

Table 1. Summary of phase shift data of the different strains of mice in experiment 2. The four columns present average phase shifts (with s.e.m. and $\mathrm{n}$ in parenthesis) for the whole circadian cycle (InT $0-24)$, the Delay region (D, InT 18 - 02), the Advance region (A, $\operatorname{InT} 02-10)$, and the dead zone (InT $10-18)$. ANOVA results indicate the statistical significance of variance among strains. In the case of the mPer mutant strains post hoc pair wise comparisons are made by Tukey tests. 


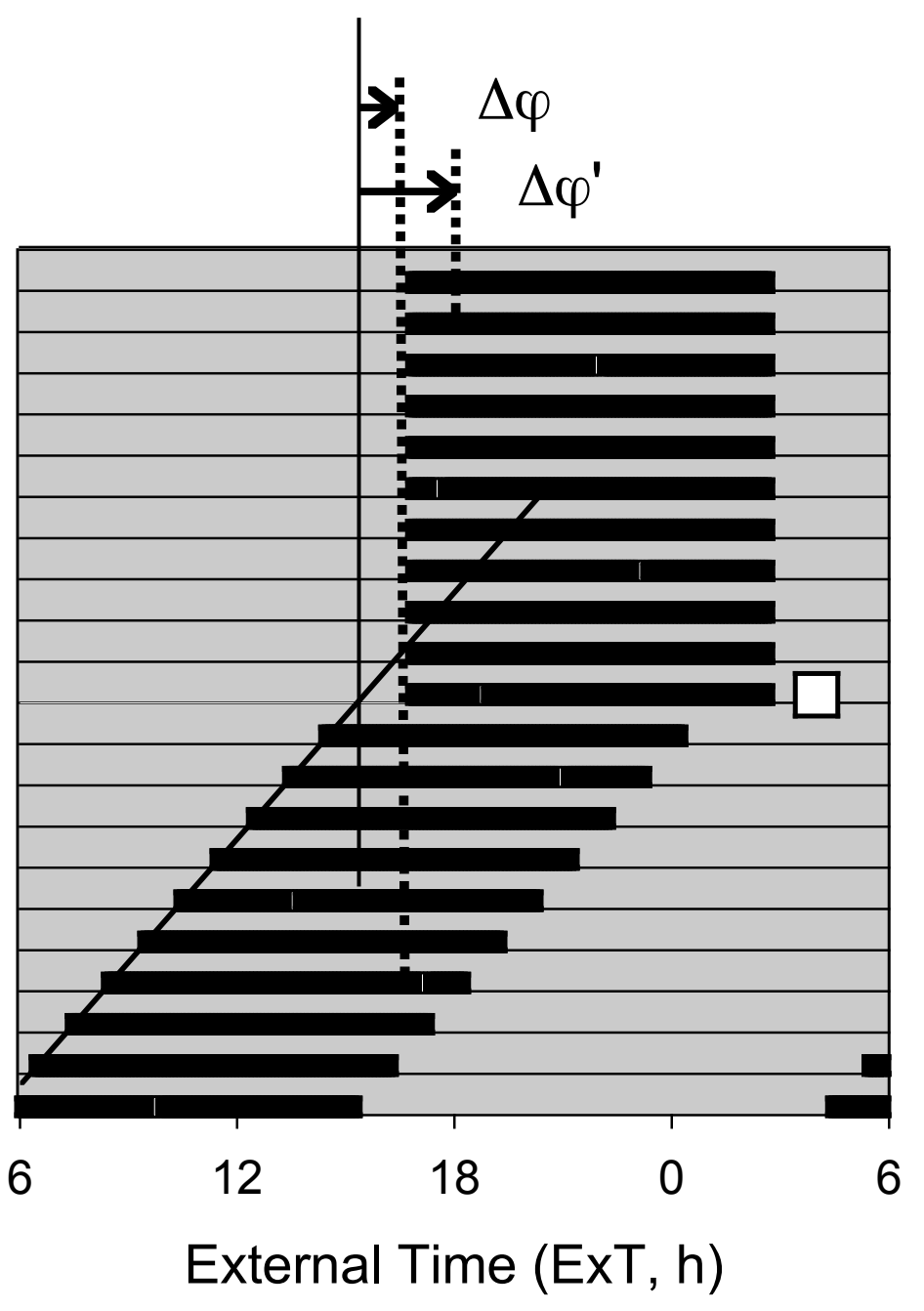

Fig. 1 


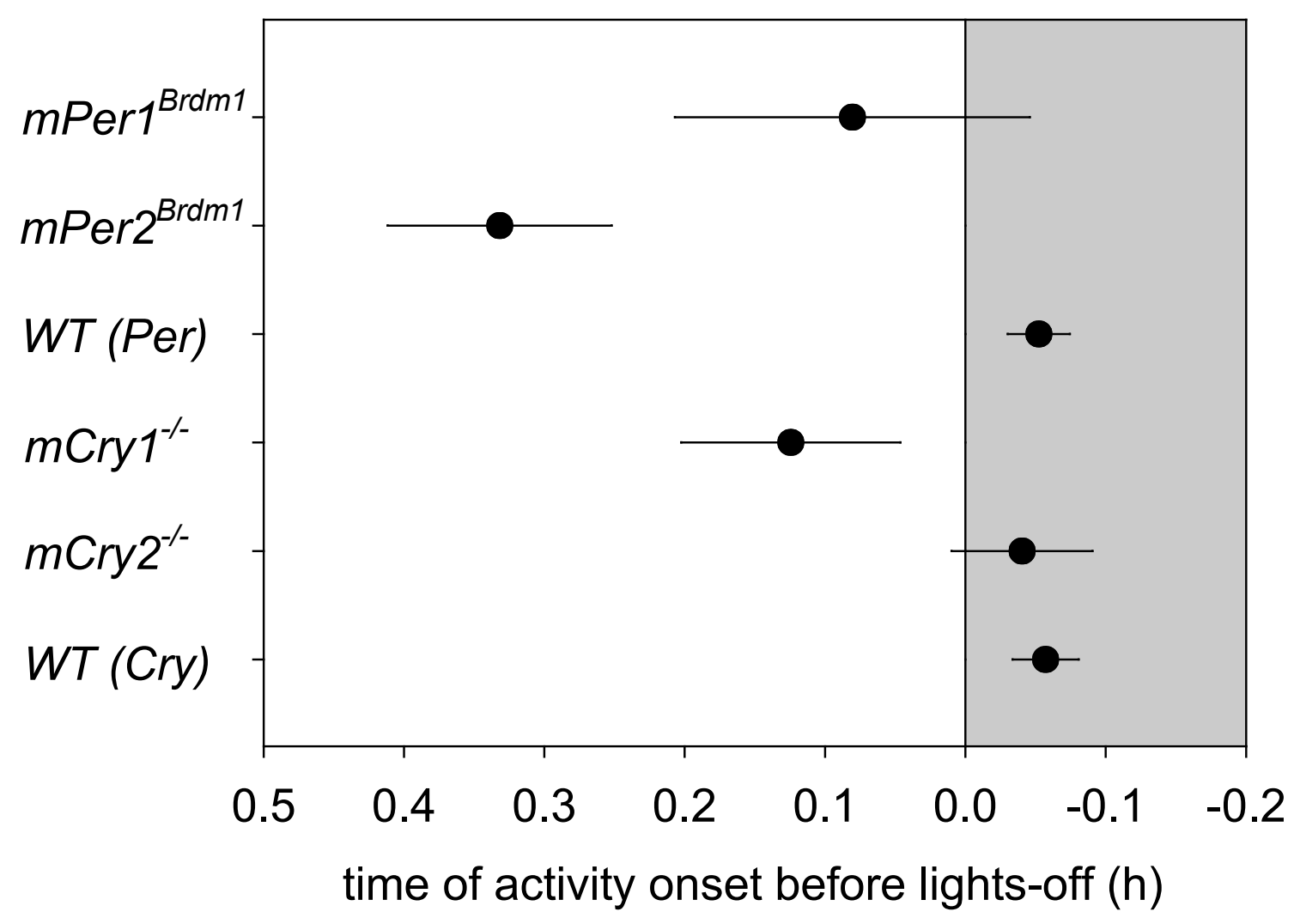

Fig. 2 


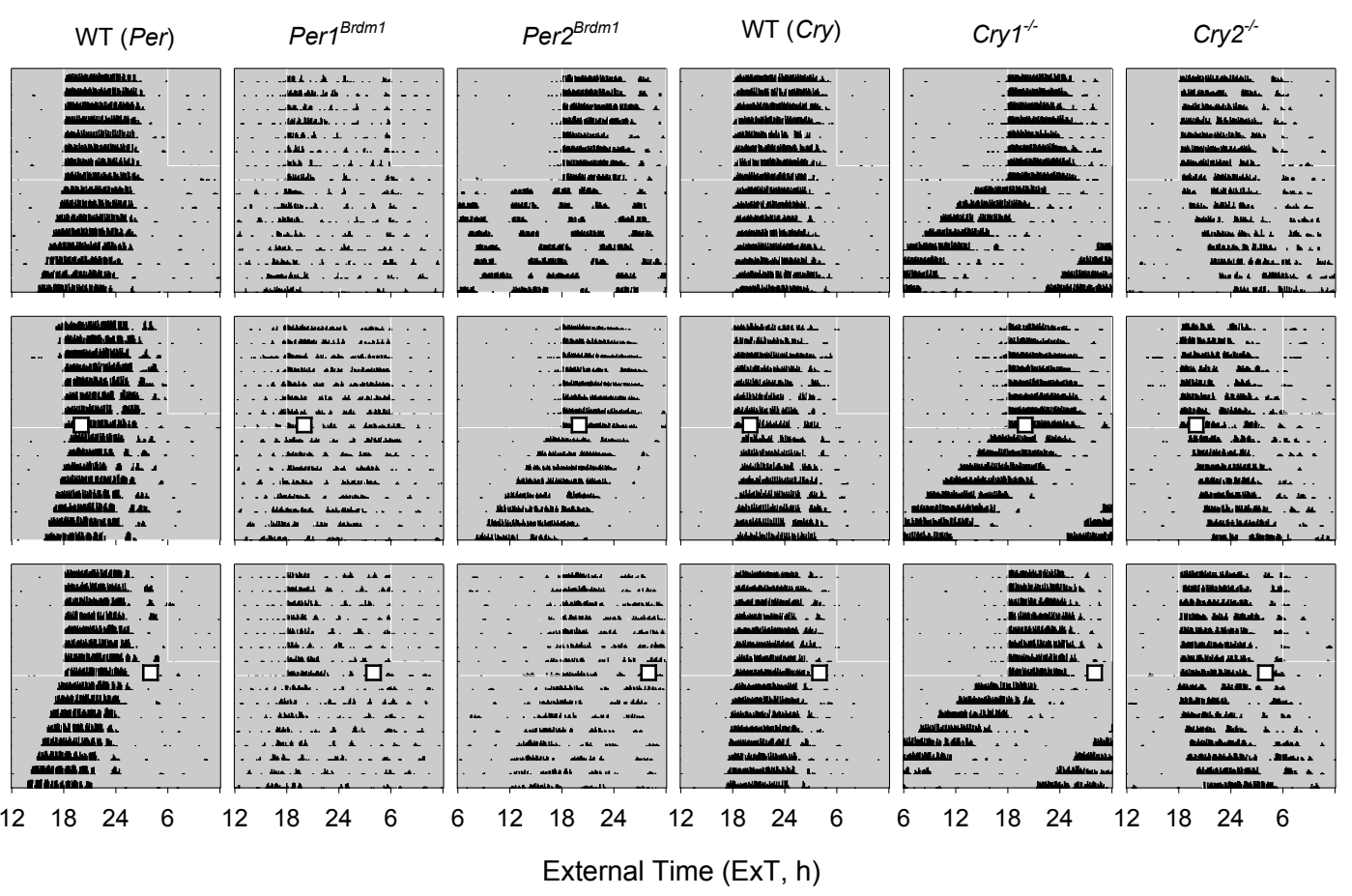

Fig. 3 

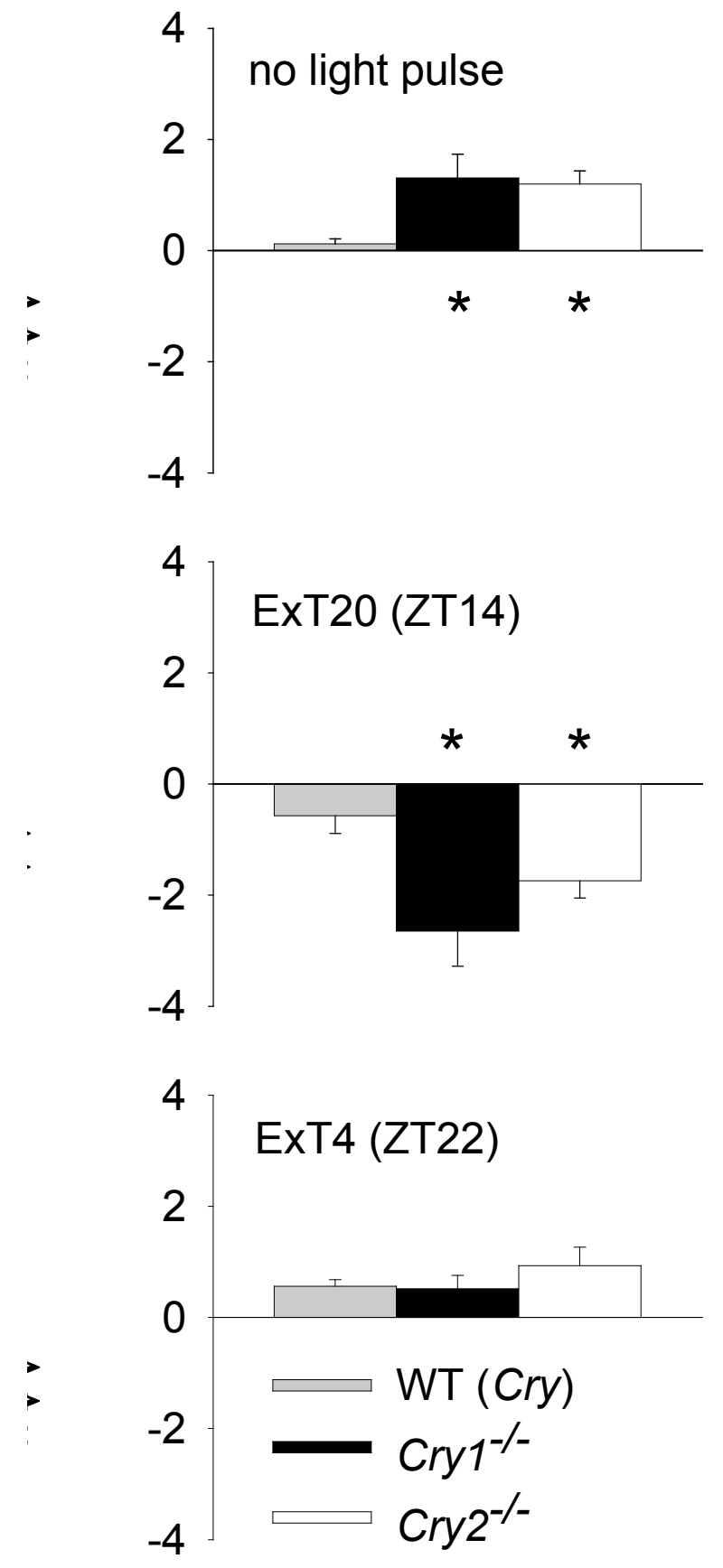

Fig. 4 


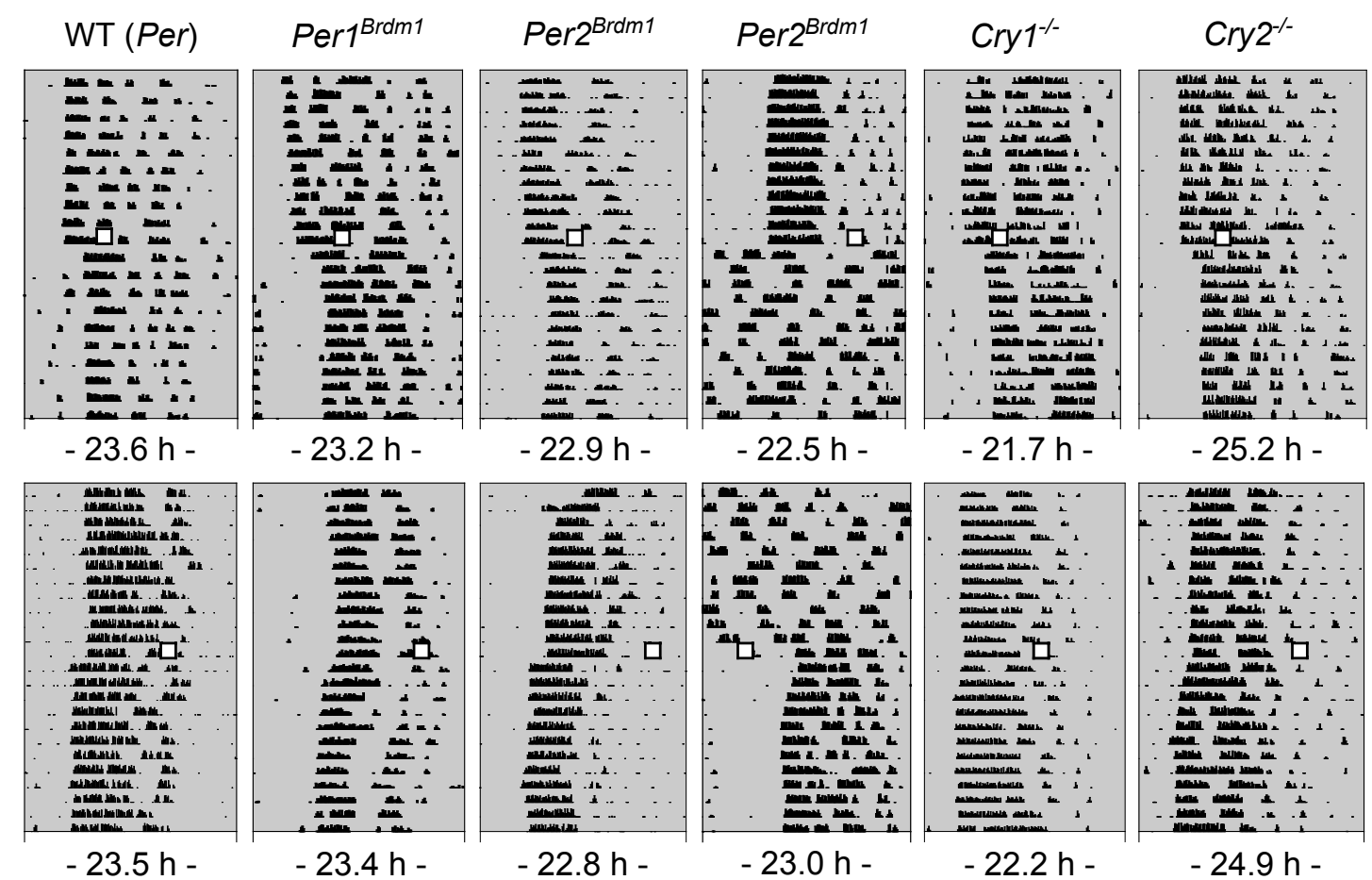

Fig. 5 

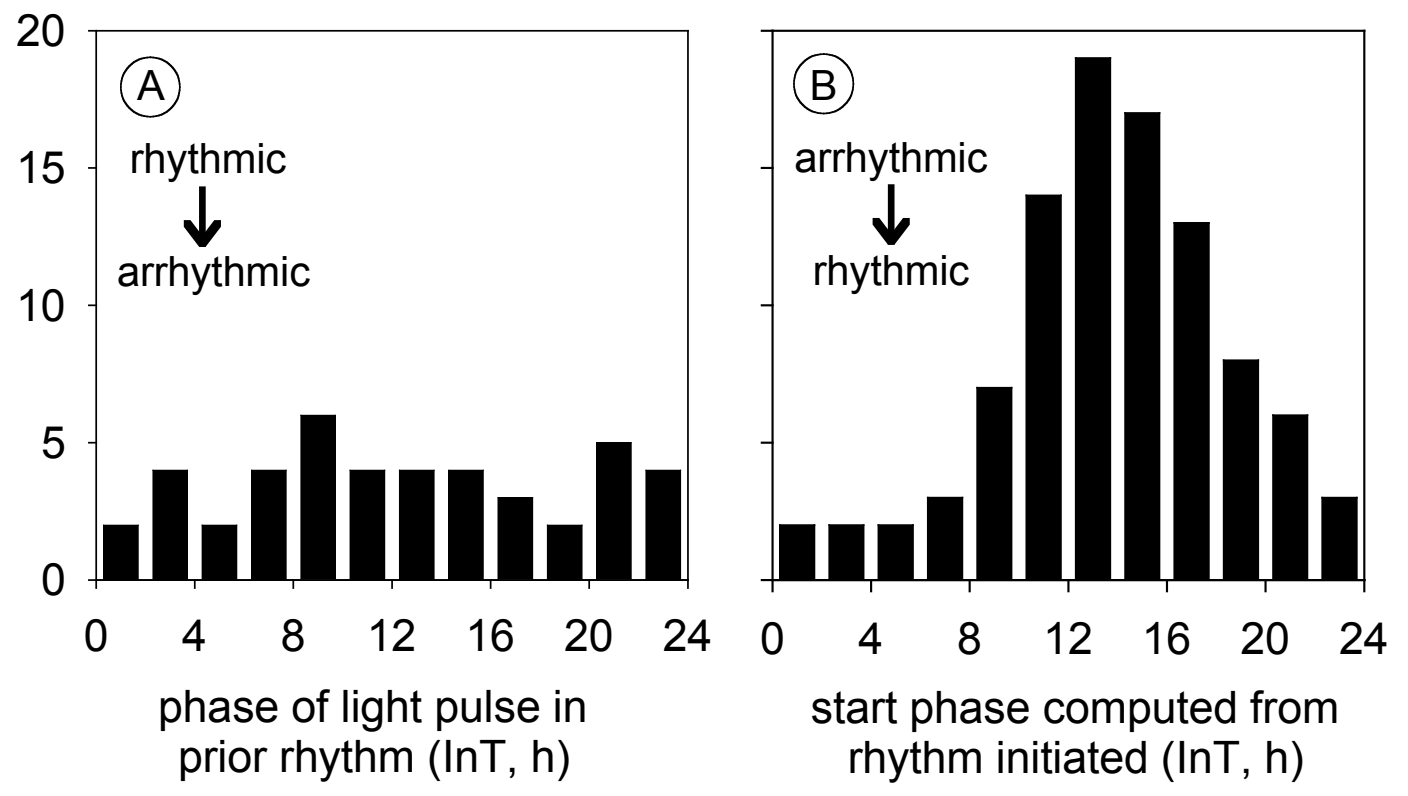

Fig. 6 


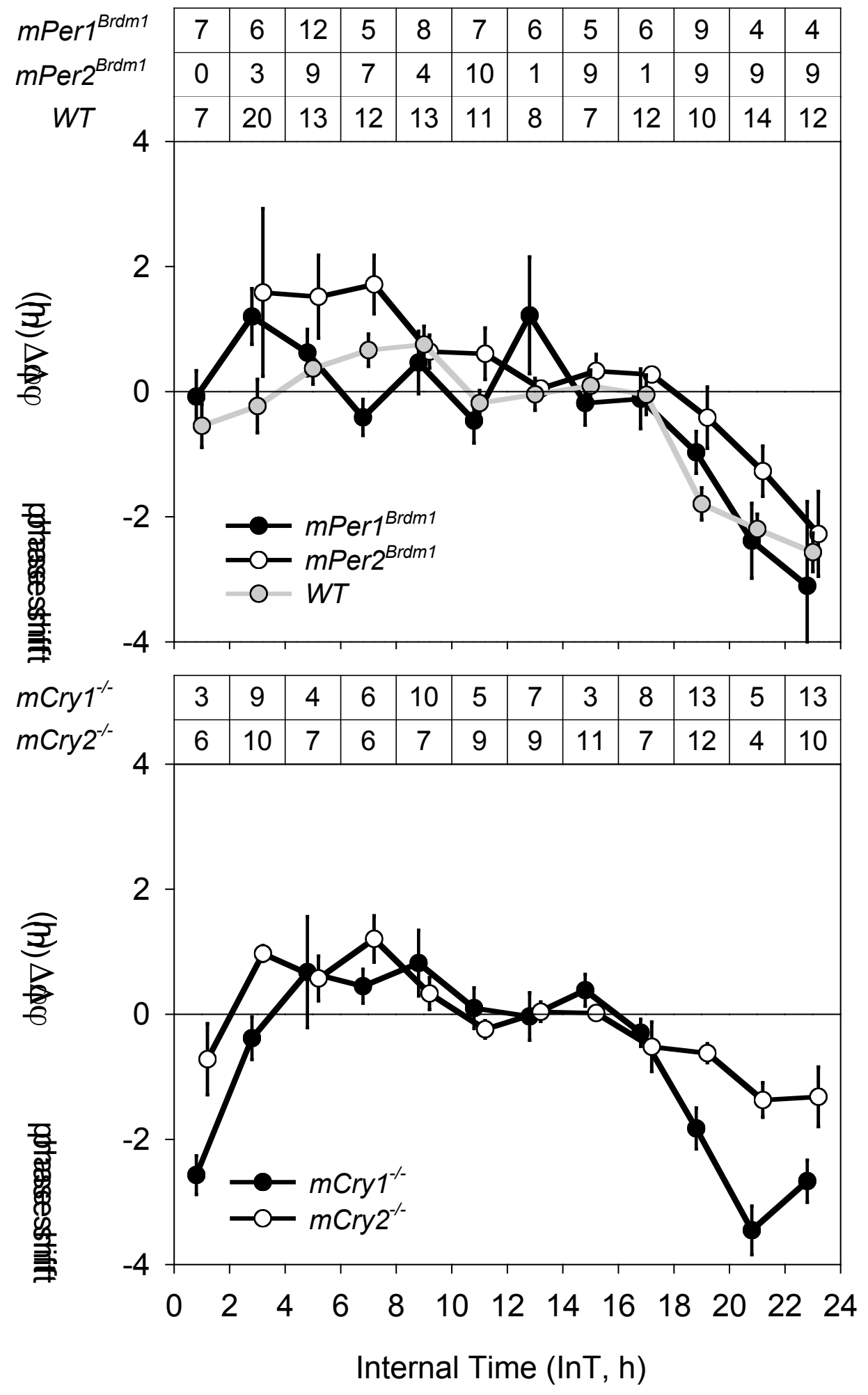

Fig. 7 


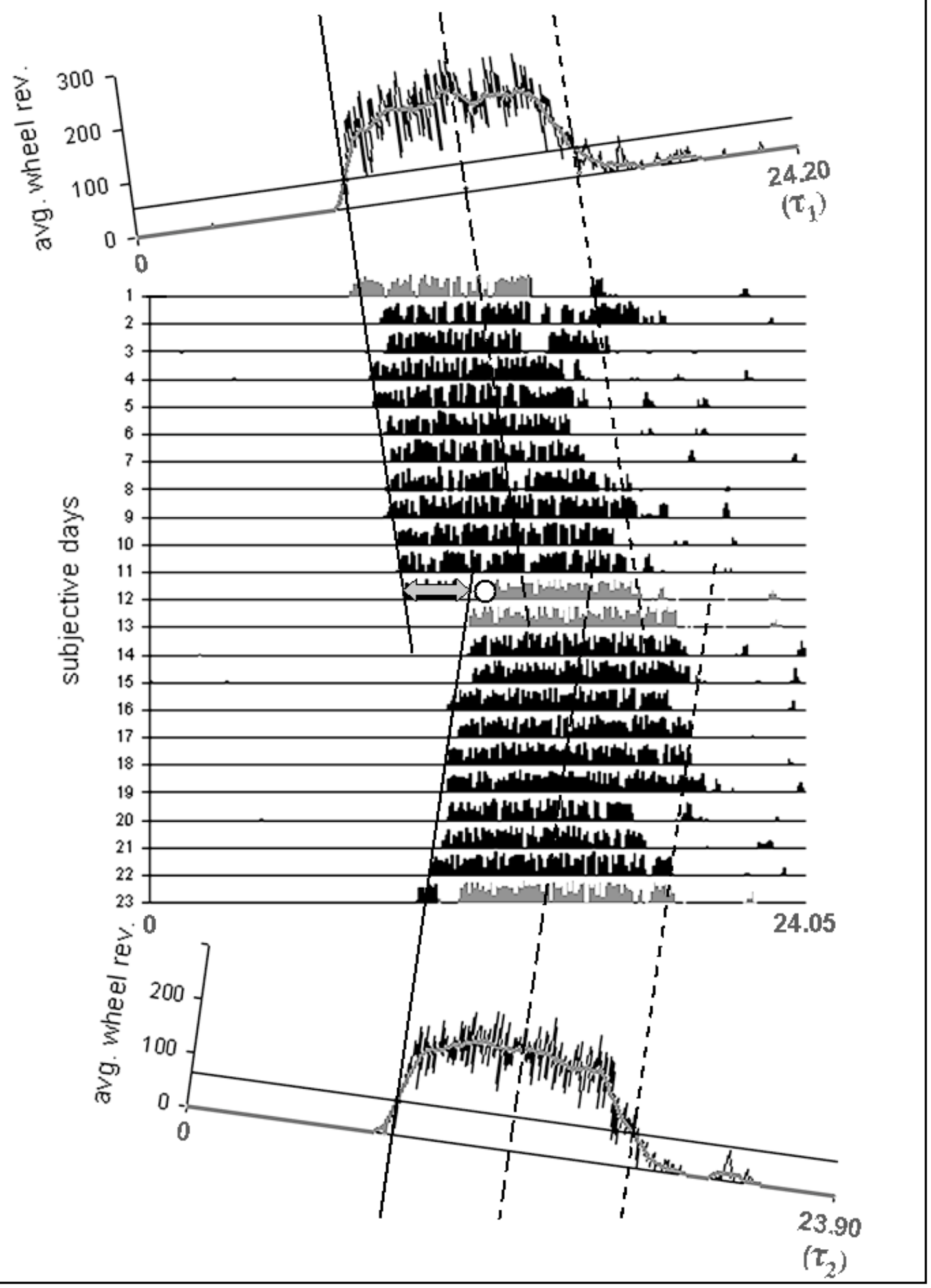

Fig. 8 